Although it is difficult to envisage a model that can account for all the differing evidence now in the literature, this idea, unlike that proposed in Fig. 1b, would be consistent with both the data from Dictyostelium and the new work from Innocenti et al..

Of course, there is still much to learn about the individual components of the WAVE regulatory complex, particularly HSPC300, which has only recently been shown to have a crucial function in plant cytoskeletal remodelling $^{14}$. It is probable that the field will change rapidly as new insights emerge.
Indeed, the numerous interactions between the WAVE complex members and other signalling molecules or adaptors (for example Abl and Nck, among many) suggest that we may only be looking at a small part of the overall picture.

1. Pollard, T. \& Borisy, G. Cell 112, 453-465 (2003).

2. Higgs, H. D. \& Pollard, T. P. J. Cell Biol. 150 , 1311-1320 (2000).

3. Machesky, L. M. \& Insall, R. Curr. Biol. 8, 1347-1356 (1998).

4. Eden, S. et al. Nature 418, 790-793 (2002).

5. Innocenti, M. et al. Nature Cell. Biol. 6, 319-327 (2004).
6. Steffen, A et al. EMBO J. 23, 749-759 (2004).

7. Bear, J. E., Rawls, J. F. \& Saxe, C. L. J. Cell Biol. 142, 1325-1335 (1998).

8. Zallen, J. A. et al. D. J. Cell Biol. 156, 689-701 (2002).

9. Biyasheva, A. et al. J. Cell Sci. 117, 837-848 (2004).

10. Blagg, S. L., Stewart, M., Sambles, C. \& Insall, R. H. Curr. Biol. 13, 1480-1487 (2003).

11. Bogdan, S. \& Klambt, C. Development 130, 4427-4437 (2003)

12. Kunda, P., Craig, G., Dominguez, V. \& Baum, B. Curr. Biol. 13, 1867-1875 (2003).

13. Rogers, S. L., Wiedemann, U., Stuurman, N. \& Vale, R. D. J. Cell Biol. 162, 1079-1088 (2003).

14. Frank, M. J., Cartwright, H. N. \& Smith, L. G. Development 130, 753-762 (2003).

\section{Getting connected}

The ways in which cell-cell communication is achieved are many and varied: they can include indirect communication through extracellular signalling molecules, or a direct physical link such as a gap junction, an immunological or neural synapse. Reporting in the February 13 issue of Science, Hans-Hermann Gerdes and colleagues now describe a new intercellular connection - long actinbased nanotubules that form de novo between cells in culture.

They first observed these ultrafine structures using threedimensional live microscopy on PC12 cells, and then further confirmed their presence in other cell types, suggesting that this may represent a general phenomenon. These 'tunnelling nanotubes', which show a continuous membrane between connecting cells, have a diameter of 50-200 nm and can stretch over several cell diameters in length. Labelling for cytoskeletal markers reveals that they are F-actin-based, but do not contain microtubules.

How might these structures arise? Using time-lapse microscopy, the authors show that they originate from filopodialike protrusions, only one of which stabilizes after cell-cell contact. From this, and from other data such as that ruling out the possibility that they might arise from incomplete cytokinesis, the authors conclude that these structures form de novo. They then tested the idea that these nanotubules might provide a communication path between cells; indeed, unidirectional transport of organelles through these nanotubules was observed. They also found a strong correlation between organelle transfer to a neighbouring cell and the presence of a nanotube connection.

The identity of these organelles remains to be seen, but they do contain two markers of particular interest: the early endosome protein synaptophysin and the actin-based motor myosin $\mathrm{Va}$. From time-lapse microscopy analysis, the organelles appear to undergo fusion with other organelles within the receiving cells, consistent with the idea of early endosome fusion. Although the

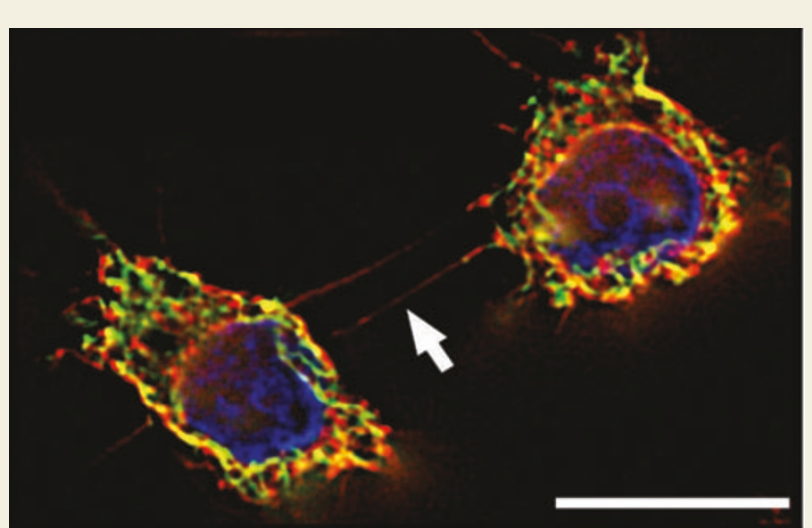

Nanotubule connections between neighbouring cells. Nanotubules (arrow) between PC12 cells contain actin (red), but not microtubules (green). Nuclei are stained in blue. Image reproduced from Rustom et al. Science 303, 1007 (2004) with permission from the American Academy of Sciences $\mathbb{0}$ (2004). Scale bar represents $15 \mu \mathrm{m}$.

exact nature of these fusion events is still unclear, organelle transfer itself seems to be an actin-dependent process that is independent of exocytosis, endocytosis and phagocytosis. Transport is also selective - although organelles can be transported, smaller soluble cytoplasmic proteins cannot.

These intriguing findings suggest that cells may have a previously unanticipated route for talking to one another. The key question now is whether these structures exist within intact tissue, and if so, for what purpose? One interesting possibility, speculated by the authors, is that they could represent a trafficking route for transcription factors or other molecular complexes, reminiscent of studies from plants.

ALISON SCHULDT 